\title{
Raynaud phenomenon causing lingual pallor and dysarthria
}

\author{
Soumya Chatterjee MD MS
}

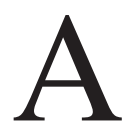

29-year-old woman presented to the rheumatology clinic with a six-month history of intermittent Raynaud phenomenon affecting her fingers (Figure 1A) and toes. She had subtle sclerodactyly but no evidence of proximal skin tightening, digital pits or ulcers, calcinosis or telangiectasias. Nailfold capillaroscopy showed dilated capillary loops but no dropout (avascular areas) or hemorrhage (Figure 1B). These clinical findings, along with a positive antinuclear antibody by immunofluorescence assay (1:640, centromere pattern), were consistent with a diagnosis of limited systemic sclerosis. ${ }^{1}$

The patient also mentioned that her tongue had been intermittently turning white or blue (Figure $1 \mathrm{C}$ and $\mathrm{D})$, associated with numbness and pronounced, but self-limiting, dysarthria during these episodes. The lingual Raynaud phenomenon tended to occur in the mornings upon awakening and during times of perceived bodily chill, generally resolving within 15-20 minutes. Occasionally, intense emotions seemed to bring on the symptoms, but just cooling the tongue (e.g., having a cold drink) did not; the patient did not report seasonal variability in their frequency.,2,3 Amlodipine $10 \mathrm{mg} / \mathrm{d}$ had slightly shortened the duration but not the frequency of the episodes.

Raynaud phenomenon results from reduced blood flow in response to cold or emotional stress, causing discoloration and paresthesias of the fingers and toes, and occasionally other parts of the body. It is frequently associated with autoimmune rheumatic diseases. Lingual Raynaud phenomenon has not been reported to be a predictor of worse prognosis from the underlying autoimmune rheumatic disease. ${ }^{3}$ Dysarthria can occur during these episodes, although no hypothesis about its mechanism has been proposed. ${ }^{2}$ Dihydropyridine calcium-channel blockers have been used successfully in the management of lingual Raynaud phenomenon. ${ }^{2}$ To facilitate diagnosis, patients who experience such transient abnormalities should be encouraged to take photographs to capture the episodes. ${ }^{4}$

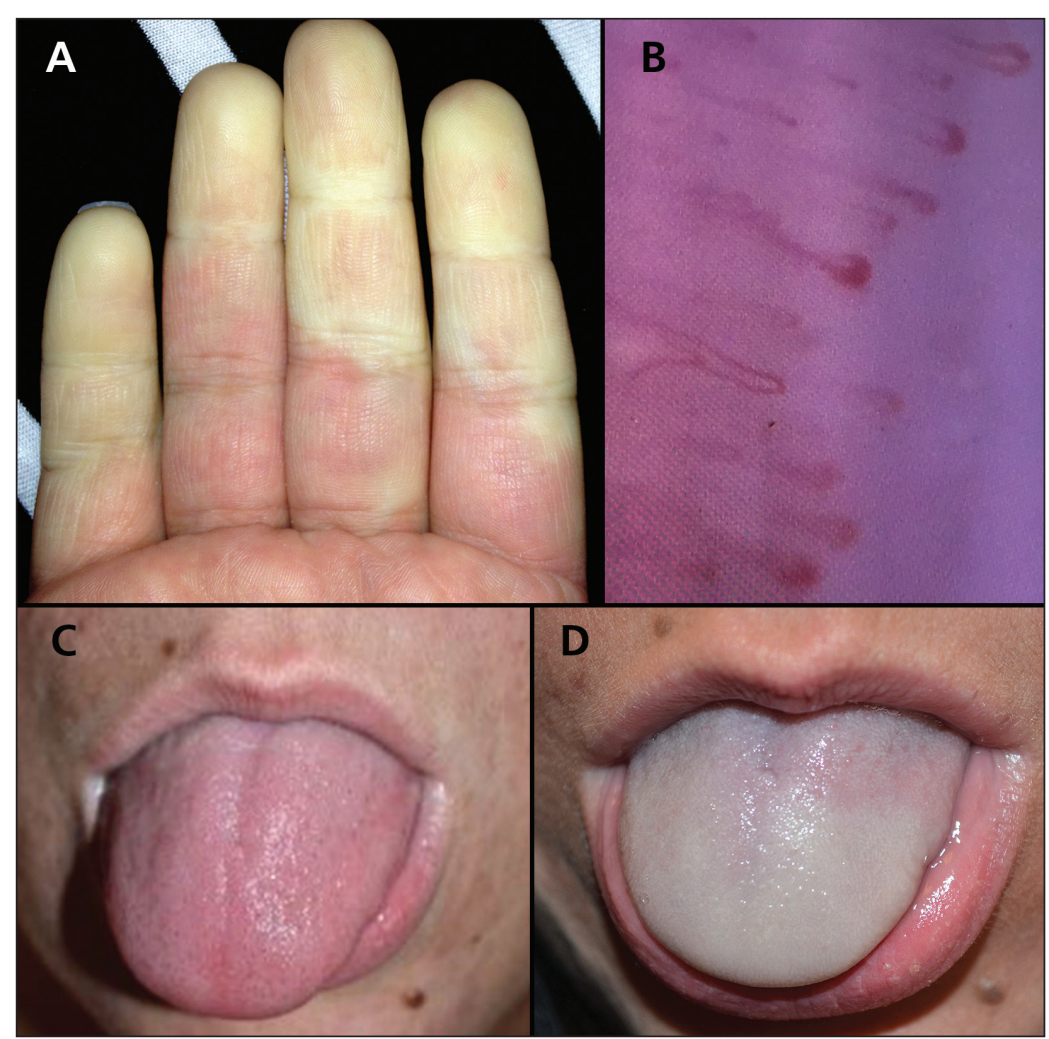

Figure 1: (A) Raynaud phenomenon affecting the patient's fingers (pallor phase). (B) Nailfold capillaroscopy showing dilated capillary loops. (C) Normal appearance of the patient's tongue. (D) Blanched white tongue during an episode of lingual Raynaud phenomenon.

\section{References}

1. van den Hoogen F, Khanna D, Fransen J, et al. 2013 classification criteria for systemic sclerosis: an American College of Rheumatology/European League against Rheumatism collaborative initiative. Arthritis Rheum 2013;65:2737-47.

2. Nielsen HV, Kristensen JK, Klemp P, et al. Paroxysmal dysarthria and Raynaud's phenomenon in the tongue. Acta Med Scand 1984;216:431-2.

3. Lioger B, Diot E. Raynaud's phenomenon of the tongue: uncommon presentation of a classical sign. QJM 2013;106:583-4.

4. Katada Y, Tanaka T. Images in clinical medicine. Lingual Raynaud's phenomenon. N Engl J Med 2012;366:e12.

Affiliation: Department of Rheumatic and Immunologic Diseases, Orthopaedic and Rheumatologic Institute, Cleveland Clinic, Cleveland, Ohio

Acknowledgement: The author thanks the patient for sharing the photograph showing her lingual Raynaud phenomenon.
Competing interests: None declared.

This article has been peer

The author has obtained patient consent.

Correspondence to:

Soumya Chatterjee, chattes@ccf.org

CMAJ 2016. DOI:10.1503 /cmaj.150547 reviewed. 